\title{
Diseño y validación de una escala de factores históricos de violencia en la niñez
}

\author{
José Ricardo Gutiérrez Quintanilla' \\ Margoth Sánchez ${ }^{2}$ \\ Ricardo Salvador Martínez ${ }^{3}$
}

Recibido: 16/07/2013 - Aceptado: 03/09/2013

\begin{abstract}
Resumen
El presente estudio tuvo como objetivo construir y validar un instrumento de medición de los factores históricos de violencia en la niñez y la juventud en El Salvador. El estudio es de tipo instrumental; se utilizó un muestreo no probabilístico, aplicándose la prueba a una muestra de 1.111 personas del área de San Salvador. Se utilizó la técnica de encuesta. La media total de edad es de 16.6 años (DT = 1.61); la muestra de mujeres fue de $563(50,60 \%)$ y la de hombres de $548(49,30 \%)$. En los resultados se tiene que la escala construida goza tanto de validez de contenido, de validez de constructo y de validez de criterio, como de fiabilidad en El Salvador.
\end{abstract}

\section{Palabras clave}

Violencia infantil, abuso del niño, factores de riesgo psicosociales-historia, El Salvador-aspectos sociales.

\section{Abstract}

The present study aims to design and validate a questionnaire for measuring the historical factors of violence in childhood and youth in El Salvador. The study is an instrumental type, with the use of a non-probability sampling. The test sample consisted of 1,111 people in the area of San Salvador, using the survey technique. The overall mean age was 16.6 years, with a standard deviation of 1.61; the sample was composed of 563 women $(50.60 \%)$ and 548 men $(49.30 \%)$. As a result of the research conducted, it shows that the scale built enjoys both content validity, construct validity and criterion validity, as well as reliability for the country of El Salvador.

\section{Keywords}

Violence against children, childhood violence child abuse, psychosocial risk factors-El Salvador history-social issues.
El comportamiento violento cruza constantemente las fronteras entre el individuo, la familia, la comunidad y la sociedad (Malvaceda-Espinoza, 2009). A su vez, sus consecuencias abarcan estos ámbitos (Unicef, 2006). Por tanto, teniendo en cuenta que la violencia no puede ser explicada por factores aislados debido a que es el resultado de un sistema, se considera importante el aporte de Bronfenbrenner (1987), quien afirma que la violencia es el resultado de la acción recíproca de factores individuales, relacionales, comunitarios, sociales y temporales (enfoque ecológico). Perspectiva asumida por la OMS (2003). Los factores de riesgo y los factores de protección son una eficaz estrategia de prevención de comportamientos antisociales en los adolescentes; parte de identificar los factores de riesgo que influyen en esos comportamientos. Pero, a la vez, al comprobarse que existen también unos factores protectores que impiden a algunos jóvenes y adolescentes tener comportamientos antisociales. A pesar de encontrarse en claras situaciones de riesgo, el fortalecer o potenciar estos factores protectores tendrá también

\footnotetext{
José Ricardo Gutiérrez Quintanilla. Es Doctor en Psicología. Profesor, investigador. Universidad Tecnológica de El Salvador. jose.gutierrez@utec.edu.sv.

Ricardo Salvador Martínez. Es licenciado en Psicología, Co-investigador. Docente Academia Nacional de Seguridad Pública. ricardo.martinez@ansp.gob.sv.
} 
importancia para reducir la delincuencia juvenil. Los factores protectores se pueden clasificar, siguiendo a Howell (1997), en tres grupos: factores protectores individuales, donde se incluyen: género femenino, alta inteligencia, habilidades sociales, locus de control interno (Garrido y López, 1995), o temperamento resistente; vínculos sociales que incluyen: afectividad, apoyo emocional o buenas relaciones familiares; y creencias saludables y sólidos modelos de comportamiento, que incluyen: aprendizaje de normas y valores sólidos, compromiso con los valores morales y sociales, buenos modelos de referencia. Los modelos 0 estrategias de prevención pueden sustentarse en ambos modelos teóricos, para procurar eliminar o disminuir los factores de riesgo, o bien incrementar y potenciar los factores de protección. Si los factores de riesgo pueden ser disminuidos y los factores protectores incrementados por una acción preventiva, entonces las probabilidades de reducir la delincuencia y la violencia juvenil se verá incrementada (Howell, 1997; Hawkins et al., 2000).

Las características individuales (de personalidad) como los contextos familiar, relacional, comunitario, social y las condiciones estructurales donde se desarrolla y convive el joven, pueden ser calificados como factores de riesgo y de protección. Dependerá de la estabilidad, el equilibrio, la armonía y los valores de cada ámbito implicado. Lo anterior indica que los desajustes, la inestabilidad, la falta de control y armonía en cada dimensión finalizará convirtiéndose en un factor de riesgo de las conductas desviadas, como la agresividad, el consumo de drogas y alcohol; alteraciones de la personalidad como: las conductas antisociales, inestabilidad emocional, la ansiedad, la depresión y actos delictivos. Debido a que en este estudio se construirá un instrumento que evalúe los factores históricos de riesgo de violencia juvenil, es fundamental describir qué variables serán consideradas como factores de riesgo de violencia en la niñez y juventud.

Hawkins (1985) considera como factores de riesgo a "cualquier circunstancia o evento de naturaleza biológica, psicológica o social, cuya presencia o ausencia modifica la probabilidad de que se presente un problema determinado en una persona o comunidad". Para Hawkins, los factores de protección son aquellos factores psicológicos y sociales que modifican la acción de un factor de riesgo para desestimular o evitar la aparición de la problemática. Son los recursos personales o sociales que atenúan o neutralizan el impacto del riesgo. Clayton (1992) entiende por factor de riesgo "un atributo o característica individual, condición situacional y contexto ambiental que incrementa la probabilidad de un comportamiento violento y de consumo de drogas. Asimismo, este autor entiende por factor de protección a "un atributo o característica individual, condición situacional y contexto ambiental que inhibe, reduce 0 atenúa la probabilidad de una acción violenta, uso y abuso de drogas". Por otra parte, Hawkins, Herrenkohl, Farrington, Devon-Brewer, Catalano, Harachi... Lynn (2000), en sus estudios, también plantean que las evidencias revisadas indican que el comportamiento violento es el resultado de las interacciones de los factores contextuales, individuales y situacionales. Estos planteamientos están en la misma sintonía que lo planteado por Luengo-Martín y Tavares-Filho (1997) y Moncada Bueno (1997). Existen diferencias casi imperceptibles en los planteamientos teóricos y empíricos de distintos autores sobre la clasificación de los factores de riesgo de violencia juvenil y de la conducta desviada en los jóvenes. Hay un alto grado de coincidencia en plantear tres escenarios básicos como grandes contextos generadores de la conducta desviada de comportamientos violentos y consumo de drogas y alcohol. Entre estos contextos coincidentes se tienen: el individual o personal, el sociocontextual y el estructural.

En el campo de la prevención de la violencia juvenil, uno de los principales problemas es la inexistencia de instrumentos para evaluar los factores de riesgo de incurrir en conductas antisociales de los jóvenes. Para responder a este problema se adoptó parte del modelo teórico de Borum, Bartel y Forth (2003), quienes plantean que los factores de riesgo y de protección de la violencia tienen cuatro componentes: factores históricos de violencia, factores individuales, factores sociales y contextuales, y factores de protección. En el presente estudio se operativizaron los factores históricos de violencia con el objetivo de construir, diseñar y validar una escala para evaluar las dimensiones históricas de violencia en la niñez, es decir, los tipos de violencia sufridos en la niñez. Producto de este proceso se obtuvieron las dimensiones siguientes: víctima de violencia psicológica y familiar, supervisión y control de los padres, víctima de violencia física, violencia física ejercida, ambiente familiar hostil y conflicto emocional. Se aplicó la escala de Autoconcepto AF5 (García y Musitu, 1999) para obtener evidencias empíricas de validez de criterio. El presente estudio es de tipo instrumental (Montero y León, 2007).

\section{Método}

\section{Participantes}

En el presente estudio el muestreo fue no probabilístico de tipo intencionado con una media de sujetos por ítem o reactivo de 8.6. Participaron en la evaluación 1.111 jóvenes de ambos géneros; el diseño fue retrospectivo y 
transeccional; se utilizó la técnica de la encuesta. La media de edad total fue de 16.6 años $(D T=1.61)$; la muestra de mujeres fue de $563(50,60 \%)$, con una media de edad de $16.5(D T=1.58) ; y$ la de hombres de $548(49,30 \%)$, con una media de edad de $16.6(D T=1.65)$. También se midieron variables como: año que estudia, con quién vive y si realiza alguna actividad laboral.

\section{Instrumentos}

Escala de factores históricos de violencia en la niñez. Esta escala fue diseñada para medir los tipos de violencia sufrida en la infancia y en la actualidad de los jóvenes. Para analizar la validez convergente de la prueba se utilizó el instrumento Autoconcepto, forma AF5. (García y Musitu, 1999). Este cuestionario está constituido por 30 ítems divididos equitativamente en cinco dimensiones del autoconcepto: académico-laboral, social, emocional, familiar y físico.

\section{Procedimiento}

Tras un proceso de planificación y gestión administrativa con los centros educativos, se inicia la fase de aplicación de pruebas. Al momento de abordar a los estudiantes, los evaluadores cumplían la siguiente rutina: presentación personal con identificación visible; se explicaba a los estudiantes sobre el trabajo que se estaba haciendo y se solicitaba la colaboración para responder un cuestionario anónimo. Se procedía leyendo la introducción, los datos generales y se les explicó las opciones de respuestas. El evaluador insistía en la importancia del estudio, sobre la anonimidad de la prueba y la sinceridad en las respuestas. De parte de las instituciones educativas seleccionadas, se recibió una apertura y colaboración para la aplicación del instrumento. Después de la primera jornada de aplicaciones, el equipo se reunió con el director del proyecto con la intención de revisar cada una de las pruebas aplicadas y descartar aquellas que presentaban, por ejemplo, más de cinco ítems en blanco o si durante la aplicación se observaba estudiantes distraídos o desmotivados y había sospechas en el patrón de respuesta (aquiescencia). Al final de la revisión, se descartaron 55 pruebas. El paso siguiente fue iniciar el procesamiento y tabulación de las pruebas en la base de datos creada en el paquete estadístico SPSS para Windows, versión 19.0. Luego se hicieron los análisis exploratorios de rutina y los análisis finales de salida.

\section{Resultados}

Tras la revisión de diez expertos (validez de contenido) del conjunto de reactivos y la prueba piloto en 250 jóvenes, se introdujeron los cambios sugeridos a la prueba inicial.
La tercera versión de la escala de los factores históricos de violencia en la niñez (Eshivi) estaba estructurada en cinco subescalas: violencia física en el pasado, violencia psicológica, ambiente de violencia familiar, supervisión y control, y disrupción temprana de los padres. La primera subescala o dimensión estaba conformada por ocho ítems, la segunda con doce reactivos, la tercera con nueve ítems, la cuarta con diez ítems y la quinta con seis ítems. A continuación se presentan los análisis psicométricos de esta primera escala, que evalúa el factor histórico de violencia juvenil, o las experiencias pasadas de violencia. En otras palabras, esta prueba mide la victimización en la infancia del joven.

En la primera fase de este proceso, se realizó un análisis factorial exploratorio (AFE) mediante el método de componentes principales. Tanto el análisis Kaiser-MeyerOlkin $(K M O=.91)$ como la prueba de esferidad de Bartlett $\left(X^{2}(666)=12577.5, p<.001\right)$ indicaron la adecuación de los datos para este tipo de análisis. Siguiendo el procedimiento de factores con rotación Varimax, resultó en una solución de seis factores que explican el $48,74 \%$ de la varianza, presentando todos los ítems valores de saturación superiores a 0.40; a excepción de los reactivos 120, 121, 124, 125 y 127 (Mis padres han permitido que yo hiciera lo que quisiera; Solía pasar horas jugando fuera de casa y sin que mis padres lo notaran; La mayor parte de mi niñez he estado bajo el cuidado de mi madre; Mis padres me dejaron bajo el cuidado de un pariente cercano; Disfruto tener en casa a uno o ambos padres), que tuvieron una saturación inferior a 0.40 , por lo que fueron eliminados. En la tabla 1 se presenta la solución factorial obtenida y el porcentaje de la varianza explicada por cada factor. Como puede apreciarse, el análisis de componentes principales modificó la propuesta original de cinco factores (violencia física en el pasado, violencia psicológica, ambiente de violencia familiar, supervisión y control, y disrupción temprana de los padres). Pensando en este modelo, se construyó y diseño esta escala. La solución factorial nos llevó a la configuración de seis factores, $y$, debido a esta nueva estructura, en la mayoría de dimensiones se modificó o cambió el nombre de las subescalas, también hubo ítems que se reagruparon. En este sentido, se describe cada una de las dimensiones o nuevos factores de la escala: factor uno: Víctima de violencia psicológica y familiar (ítems 100, 101, 102, 103, 104, 105, 106, 107, 109, 110, 111); factor dos: Supervisión y control de los padres (ítems 115, 116, 117, 118, 119, 122, 123); factor tres: Víctima de violencia física, (ítems 96, 97, 98, 99, 108); factor cuatro: Violencia física ejercida (ítems 92, 93, 94, 95); factor cinco: Ambiente familiar hostil (ítems 112, 113, 114), y factor seis: Conflicto emocional (ítems 126, 128). 
Tabla 1.

Matriz de componentes principales (factores), su carga factorial rotada y el porcentaje de varianza explicada por cada dimensión histórica de violencia en la niñez (victimización)

\begin{tabular}{|c|c|c|c|c|c|c|}
\hline & & & TORES/COMPOI & TES & & \\
\hline & $\begin{array}{c}\text { FACTOR } 1 . \\
\text { Víctima de violencia } \\
\text { psicológica } \\
\text { y familiar }\end{array}$ & $\begin{array}{l}\text { FACTOR } 2 . \\
\text { Supervisión y } \\
\text { control de los } \\
\text { padres }\end{array}$ & $\begin{array}{c}\text { FACTOR } 3 . \\
\text { Víctima de } \\
\text { violencia física }\end{array}$ & $\begin{array}{c}\text { FACTOR } 4 . \\
\text { Violencia física } \\
\text { ejercida }\end{array}$ & $\begin{array}{c}\text { FACTOR } 5 . \\
\text { Ambiente } \\
\text { familiar hostil }\end{array}$ & $\begin{array}{c}\text { FACTOR } 6 . \\
\text { Conflicto } \\
\text { emocional }\end{array}$ \\
\hline a92 (1) & . & & & .581 & & \\
\hline a93 (2) & & & & .719 & & \\
\hline a94 (3) & & & & .684 & & \\
\hline a95 (4) & & & & .662 & & \\
\hline a96 (5) & & & .523 & & & \\
\hline a97 (6) & & & .621 & & & \\
\hline a98 (7) & & & .750 & & & \\
\hline a99 (8) & & & .446 & & & \\
\hline a100 (9) & .670 & & & & & \\
\hline a101 (10) & .729 & & & & & \\
\hline a102 (11) & .777 & & & & & \\
\hline a103 (12) & .746 & & & & & \\
\hline a104 (13) & .598 & & & & & \\
\hline a105 (14) & .651 & & & & & \\
\hline a106 (15) & .576 & & & & & \\
\hline a107 (16) & .622 & & & & & \\
\hline a108 (17) & & & .381 & & & \\
\hline a109 (18) & .682 & & & & & \\
\hline a110 (19) & .557 & & & & & \\
\hline a111 (20) & .551 & & & & & \\
\hline a112 (21) & & & & & .697 & \\
\hline a113 (22) & & & & & .648 & \\
\hline a114 (23) & & & & & .641 & \\
\hline a115 (24) & & .636 & & & & \\
\hline a116( 25) & & .723 & & & & \\
\hline a117 (26) & & .768 & & & & \\
\hline a118 (27) & & .644 & & & & \\
\hline a119 (28) & & .677 & & & & \\
\hline a122 (29) & & .519 & & & & \\
\hline a123 (30) & & .485 & & & & \\
\hline a126 (31) & & & & & & .843 \\
\hline a128 (32) & & & & & & .820 \\
\hline $\begin{array}{l}\text { \% varianza } \\
\text { explicada }\end{array}$ & 24,78 & 7,6 & 4,83 & 4,41 & 3,68 & 3,44 \\
\hline 口 & .90 & .78 & .73 & .67 & .76 & .70 \\
\hline
\end{tabular}


A continuación se realiza el análisis de ítems y de consistencia interna de los seis factores obtenidos mediante el análisis de componentes principales, incluyéndose en la estructura factorial los ítems de cada subescala. Esta se conforma del factor 1: Víctima de violencia psicológica y familiar (ítems $100,101,102,103,104,105,106,107,109,110,111)$; factor 2: Supervisión y control de los padres (ítems 115, 116, 117, $118,119,122,123) ;$ factor 3 : Víctima de violencia física (ítems 96, 97, 98, 99, 108); factor 4: Violencia física ejercida (ítems 92, 93, 94, 95); factor 5: Ambiente familiar hostil (ítems 112, $113,114)$, y factor 6: Conflicto emocional (ítems 126, 128). En la tabla 5 se puede observar que existe una correlación significativa entre las dimensiones o subescalas de la prueba.

La subescala Víctima de violencia psicológica y familiar tuvo un valor de consistencia interna alfa de Cronbach de .90. La media de respuesta a los ítems osciló entre 1.18 (ítem 106) y 1.87 (ítem 100). En la mayoría de los ítems, la media se encuentra un poco por debajo de la media de la escala, que es de 2.5. Las desviaciones típicas en la mayoría de los ítems están cercanas a la unidad. Junto a estos estadísticos se calculó la correlación ítem-total corregida y el alfa de Cronbach, si se elimina el ítem; las correlaciones ítem-total corregidas se situaron por encima de .51; y en ningún caso la eliminación de algún ítem suponía el incremento del valor alfa de Cronbach.

En la subescala Supervisión y control de los padres, la consistencia interna alfa de Cronbach obtenida fue de .78. La media de respuesta a los ítems osciló entre 2.66 (ítem 115) y 3.07 (ítem 122). En todos los ítems la media se encuentra próxima a la media de la escala, que es 2.5. La desviación típica en la mayoría de los ítems es de 1; a excepción del ítem 118, que es de .97. Junto a estos estadísticos se calculó la correlación ítem-total corregido y el alfa de Cronbach, si se elimina el ítem; las correlaciones ítems total corregidas se situaron por encima de 0.40; y en ningún caso la eliminación de algún ítem suponía el incremento del valor alfa de Cronbach.

En la subescala Víctima de violencia física la consistencia interna alfa de Cronbach obtenida fue de .73. La media de respuesta a los ítems osciló entre 1.19 (ítems 97, 98) y 1.58 (ítem 99). En todos los ítems la media se encuentra un poco por debajo de la media de la escala, que es de 2.5. La desviación típica en la mayoría de los ítems, los valores se encuentran relativamente por debajo de la unidad. En conjunto con estos datos se calculó la correlación ítems total corregido y el alfa de Cronbach, si el ítem es eliminado; las correlaciones ítem total corregidas se situaron por encima de .43; y en ningún caso la eliminación de algún ítem suponía el incremento del valor alfa de Cronbach.

En la subescala Violencia física ejercida, la consistencia interna alfa de Cronbach obtenida fue de .67. La media de respuesta a los ítems osciló entre 1.59 (ítem 95) y 2.20 (ítem 92). En todos los ítems la media se encuentra un poco por debajo de la media de la escala, que es de 2.5. La desviación típica en dos ítems los valores se encuentran en la unidad, y en dos ítems están cercanos a la unidad. En conjunto con estos datos se calculó la correlación ítem total corregido y el alfa de Cronbach, si el ítem es eliminado; las correlaciones ítems total corregidas se situaron por encima de .41; y en ningún caso la eliminación de algún ítem suponía el incremento del valor alfa de Cronbach.

En la subescala Ambiente familiar hostil, la consistencia interna alfa de Cronbach obtenida fue de .76. La media de respuesta a los ítems osciló entre 1.52 (ítem 114) y 1.88 (ítem 113). En todos los ítems la media se encuentra un poco por debajo de la media de la escala, que es de 2.5. La desviación típica en un ítem el valor se encuentra en la unidad, y en dos ítems está cercano a la unidad. En conjunto con estos datos se calculó la correlación ítems total corregido y el alfa de Cronbach, si el ítem es eliminado; las correlaciones ítems total corregidas se situaron por encima de .53; y en ningún caso la eliminación de algún ítem suponía el incremento del valor alfa de Cronbach.

En al subescala Conflicto emocional, la consistencia interna alfa de Cronbach obtenida fue de .70. La media de respuesta a los ítems osciló entre 2.27 (ítem 128) y 2.42 (ítem 126). En los dos ítems la media se encuentra cercana a la media de la escala, que es de 2.5. La desviación típica en los dos ítems el valor se encuentra en la unidad. En conjunto con estos datos se calculó la correlación ítems total corregido y el alfa de Cronbach, si el ítem es eliminado; las correlaciones ítems total corregidas se situaron en .53; y en ningún caso la eliminación de algún ítem suponía el incremento del valor alfa de Cronbach.

Existe una correlación estadísticamente significativa entre la mayoría de las dimensiones de la Escala de factores históricos de violencia juvenil y las dimensiones de la escala de Autoconcepto AF5. Algunas correlaciones son positivas, mientras la mayoría son negativas. Estas correlaciones son relevantes para el estudio, debido a que demuestran evidencias empíricas de la fortaleza de la estructura factorial de la escala construida. En este sentido, son evidencias estadísticas de una adecuada validez convergente o de criterio de la prueba para estudiar los factores de riesgo de violencia en adolescentes salvadoreños. Indicando lo anterior que se puede utilizar para medir y evaluar esta problemática relacionada con la conducta violenta en jóvenes salvadoreños.

\section{Discusión}

Tras un proceso sistemático de revisión de pruebas y modelos teóricos de factores de riesgo y protección de violencia juvenil, el equipo investigador adoptó la estructura factorial de cuatro factores de Borum, Bartel y Forth (2003), convirtiendo sus 
componentes en subescalas o dimensiones, construyendo un número de ítems por cada dimensión, redactándose los ítems pensando en el contexto salvadoreño y en una población normal de adolescentes. La presente discusión se refiere al primer factor o factores históricos de violencia en la niñez y la juventud.

Para el análisis psicométrico de la Escala de factores históricos de violencia infantil (victimización infantil), mediante el Análisis factorial exploratorio (AFE), a través del método de componentes principales (Cortina, 1993; Comrey, 1988; Floyd y Widaman, 1995), este resultó en una solución factorial que explica el 48,74 \% de la varianza total explicada, con una solución de seis factores o subescalas, siendo estas: Víctima de violencia psicológica y familiar $(a=.90)$, Supervisión y control de los padres $(\mathrm{a}=.78)$, Víctima de violencia física $(\mathrm{a}=$ .73), Violencia física ejercida $(\mathrm{a}=.67)$, Ambiente familiar hostil $(\mathrm{a}=.76)$ y Conflicto emocional $(\mathrm{a}=.70)$. Todas las variables 0 ítems que conforman las dimensiones cumplen los criterios de una carga factorial superior a .40. Estos resultados están en sintonía con lo recomendado por Stevens (1992), quien señala que las saturaciones de los factores deben ser iguales o superiores a .40, aunque Floyd y Widaman (1995) proponen unos criterios menos restrictivos, entre .25 y .30, para muestras superiores a 300 . También cumplen los criterios de consistencia interna mediante el alfa de Cronbach que son superiores a .70; solo la subescala Violencia física ejercida presenta un alfa de .67. Por otra parte, se establecieron las correlaciones entre las subescalas y con otros criterios (validez de criterio), encontrándose una correlación significativa en ambos casos. Los criterios antes señalados llevan a la conclusión de que la escala de factores históricos de violencia infantil, integrada por seis dimensiones, es una prueba que posee las adecuadas propiedades psicométricas de validez de contenido, validez de constructo, validez de criterios y la confiabilidad para evaluar y medir los factores de riesgo de violencia infantil y juvenil en El Salvador.

\section{Referencias}

Borum, R.; Bartel, P. y Forth, A. (2003). Structured Assessment of Violence Risk in Youth. Professional Manual. Oxford: Pearson

Bronfenbrenner, U. (1979). The ecologyof Human Development. Cambridge, Harvard University Press. (Trad. Cast.): La ecología del desarrollo humano. Barcelona, Ediciones Paidós, 1987).

Clayton, R.R. (1992). Transitions in drug use: Risk and protective factors. En Glantz, M. y Pickens, R. (Eds.). Vulnerability to drug abuse (pp. 15-51). Washington, DC. American Psychological Association.

Comrey, A.L. (1988). Factor-analytic methods of scale development in personality and clinical psychology. Journal of Consulting and Clinical Psychology, 56, 754-761.

Cortina, J.M. (1993). What is coefficient alpha? An examination of theory and applications. Journal of Applied Psychology, 78, 98-104.

Floyd, F.J. y Widaman, K.F. (1995). Factor analysis in the development and refinement of clinical assessment instruments. Psychological Assessment, 7, 286-299.

Garrido, V. y López, M.J. (1995). La prevención de la delincuencia: el enfoque de la competencia social, Tirant lo Blanch, Valencia.

Hawkins, J.D.; Lishner, D. y Catalano, R., (1985). Childhood predictors and the prevention of adolescent substance abuse. En Jones, C. J., y Battjes, R. (Eds.). Etiology of drug abuse: Implications for prevention (pp. 75-126). Rockville: National Institute on Drug Abuse.

Hawkins, J.D.; Herrenkohl, T.; Farrington, D.P.; Devon-Brewer, R.; Catalano, F.; Harachi, T.W. y Cothern, Lynn (2000). "Predictors of Youth Violence", en Juvenile Justice Bulletin, April, OJJDP, Washington, DC.1-10.

Howell, J.C. (1997). Juvenile Justice \& Youth Violence, Sage, Thousand Oaks, CA.

Luengo-Martín, M.A. y Tavares-Filho, E.T. (1997). La estructura de los valores en delincuentes y no delincuentes: un análisis comparativo en los adolescentes marginados en manaus. XXVI Congreso Iberoamericano de Psicología; Sao Paulo, Brasil.

Malvaceda-Espinoza, E. (2009). Análisis psicosocial de la violencia entre el conflicto y el desarrollo social, Cuad. Difus.14, p. 26.

Moncada-Bueno, S. (1997). Factores de riesgo y de protección en el consumo de drogas, en Varios: Prevención de la drogodependencia. Análisis y propuesta de actuación. Madrid. Ministerio del Interior. Delegación del Plan Nacional sobre drogas. 85-104.

Montero, I. y León, O.G. (2007). A guide for naming research studies in Psychology. International Journal of Clinical and Health Psychology, 7, 847-862.

Unicef (2006). El Estado Mundial de la Infancia. Excluidos e invisibles. Autor. Unicef House, 3 UN Plaza, Nueva York, NY 10017, USA. Disponibles en: http://www.unicef. org/spanish/sowc06/pdfs/sowc06_fullreport_sp.pdf

Organización Mundial de la Salud (OMS), (2003). Informe mundial sobre la violencia y la salud. Washington, DC. Autor: Osterlind, S.J. (1989).

Unicef (2006). El Estado Mundial de la Infancia. Excluidos e invisibles. Autor. Unicef House, 3 UN Plaza, Nueva York, NY 10017, USA. Disponible en: http://www.unicef.org/ spanish/sowc06/pdfs/sowc06 fullreport sp.pdf

Stevens, J. (1992). Applied multivariate statistics for the social sciences. Hillsdale, NJ: Lawrence Erlbaum. 